\title{
Opioid Prescribing: How Well Do We Know Ourselves?
}

\author{
Rachel S. Wightman ${ }^{1} \cdot$ Lewis S. Nelson ${ }^{2}$
}

Received: 8 July 2016 / Revised: 8 July 2016 / Accepted: 19 July 2016 / Published online: 4 August 2016

(C) American College of Medical Toxicology 2016

Get your facts first, then you can distort them as you please.-Mark Twain

"I would rather over-prescribe and risk some medication diversion or non-medical use, than under-prescribe and not treat a patient's pain." This statement, taken from a survey published in the current issue of JMT by Pomerleau et al. 2016 [1], was used to assess clinicians' self-reported attitude about the challenge in meeting individual patient needs while balancing public health risk. The statement essentially pinpoints the current dilemma in the ED: alleviation of suffering in an individual may expose that patient or others (through misuse or diversion) to the risk of opioid analgesic (OA) abuse, addiction, overdose, and death. Pain-related complaints are the most common reason for Emergency Department (ED) visits [2, 3], and ED providers are among the top five medical specialist groups writing prescriptions for OAs in patients under 40 years of age [4]. However, only $17 \%$ of ED patients are given a prescription for an OA on discharge and the majority of prescriptions dispensed are for a small number of immediate release pills $[5,6]$. Still, iatrogenic opioid abuse and addiction remain a concern, because half of ED patients with opioid-use disorders were first exposed to an opioid by a prescription from a medical provider [7].

Responsible opioid analgesic prescribing is paramount in our efforts to combat the epidemic of opioid abuse and promote safe opioid use, but little is currently known about which factors drive decision-making among emergency department

Rachel S. Wightman

rachel.wightman@nyumc.org

1 Ronald O. Perelman Department of Emergency Medicine, New York University School of Medicine, New York, USA

2 Department of Emergency Medicine, Rutgers New Jersey Medical School, Newark, NJ, USA
(ED) prescribers of OAs. Pomerleau et al. highlights the complexity of the decision-making process and brings forth a number of interesting issues. Through a series of queries, the study explores the factors providers believe affect their OA prescribing practices, as well as their attitudes toward OA prescribing. Of course, individual provider opinions and attitudes cannot provide the complete picture. Patient age, race, type of insurance, provider training and experience, functionality of PDMPs, and geographic location are a few of the many factors highlighted in the literature to affect OA prescribing decisions.

Whether to prescribe an OA for any given patient not only depends on the aforementioned patient and provider factors and the culture of the work environment, but also on the quality and type of patient doctor communication. One study showed that when faced with identical hypothetical case scenarios, ED physicians' decisions whether to prescribe an OA varied greatly [14]. For the same clinical scenario, subtle differences in the patient-doctor interaction (for example, a patient requesting a specific drug or an additional dose of pain medication) could change the likelihood of prescribing substantially [18]. To support this unconscious bias, Pomerleau, et al. uncovered some interesting paradoxes. For example, survey respondents report that they would be less likely to write a prescription for a patient with an opioid use disorder, but they also state their skills to identify patients with opioid use disorders and determine who is doctor shopping are poor.

The emergency physician respondents in Pomerleau et al. ranked patient's overall satisfaction as a neutral factor in their decision to prescribe an opioid. Though this may be more true in the ED, patient satisfaction has a more prominent impact on the decision to prescribe an opioid in other clinical settings. For example, many hospitals collect data on the patient's experience and tie such feedback with physician payments (although CME recently announced that this may end). Although 
some EDs may have been subject to such feedback, the soonto-be implemented EDCAHPS, which collect information on a patient's pain management experience during an ED visit, will likely raise the prominence of satisfaction with pain management. While physicians report prescribing an OA to expedite patient discharge or improve patient satisfaction scores [14], other studies demonstrate no association between ED patient satisfaction scores and administration of OA medications [17]. However, it remains unclear if this apparent divergence represents a study methodological distinction, a true assessment of actual practice, or a desirable selfinterpretation of a human tendency.

None of the providers in the survey by Pomerleau et al. felt strongly that they give more opioid medications than their colleagues. The extent to which social desirability and/or reporting bias affect provider responses and the amount an individual's belief about opioid prescribing may vary from their actual practice is unknown. Provider perception versus actual practice of OA prescribing has not been studied, but in other realms, the differences between actual behavior and personal perception of one's behavior can vary substantially. For example, just $8 \%$ of physicians surveyed believed that they were susceptible to promotional items such as pharmaceutical branded pens [19]. However, the receipt of minimal gifts, valued at less than 20 dollars, was associated with an increased rate of prescribing the promoted brand-name medication relative to alternatives within the same drug class [20]. When the effect of subtle exposures to branded pharmaceutical promotional items was tested experimentally in medical students, pharmaceutical marketing was found to influence implicit attitudes of brand preference. Of interest in this same study, the effect of drug marketing was reversed among fourth-year medical students whose institution had a restrictive policy to limit pharmaceutical marketing [21]. Thus, we may not know ourselves as well as we think we do. Stated empirically, if there was not a "return on investment," industry would most assuredly end its practice.

Additionally, published federal and state guidelines for OA prescribing and/or institutional guidelines or mandates, as well as PDMPs, are widespread and affect prescribing practices $[8,9]$. However, the implementation of institutional policies varies widely. In a study of New England hospitals, policies recommending use of screening tools and prescribing naloxone for at-risk patients are uncommon, but policies regarding utilization of PDMPs and referral of patients with opioid use disorders to recovery resources are more prevalent [10]. The presence of an opioid prescribing policy has shown divergent results on practice, both reducing [11-13] and having no effect on [14] the amount and strength of prescribed OA. Other institutional efforts, such as formulary maintenance, may surreptitiously limit the range of prescribing practices. For example, the workhorse opioid that is available on the electronic medical record (EMR) may direct the opioid that is prescribed. A recent study demonstrated that changes to the default setting in the EMR from brand name to generic medications increased the prescribing of generic-equivalent medications [15]. Similarly, changes to the default setting for the dose and duration of opioid analgesic prescription in the EMR affected the prescribing of post-operative pain medication [16].

The decisions required to write an opioid prescription are complex and nuanced. Although individual attitudes are interesting and important to explore, the decision making process is larger than simply a brief encounter between an individual provider and patient. Rather, this decision varies based on the context, patient, prescriber, health care environment, and culture and may be tempered by guidelines and mandates. To be able to draw conclusions based on the data presented by Pomerleau et al., an important next step would be to pair provider beliefs and attitudes about OA prescribing with an objective measure of prescriber behavior, namely the actual prescribing practices. In order to make targeted positive changes to combat the epidemic of prescription, opioid abuse individual prescribers must clearly understand their attitudes and biases, and think and think again, in a metacognitive fashion, about their actual behaviors and practices.

\section{Compliance with Ethical Standards}

Conflicts of Interest None

Sources of Funding None

\section{Reference}

1. Pomerleau AC, Schrager JD, Morgan BW. Pilot study of the importance of factors affecting emergency department opioid analgesic prescribing decisions. J Med Toxicol 2016.

2. CDC National Hospital Ambulatory Medical Care Survey: Emergency Department. 2011.

3. Todd KH, Ducharme J, Choiniere M, et al. Pain in the emergency department: results of the pain and emergency medicine initiative (PEMI) multicenter study. J Pain. 2007;8:460-6.

4. Volkow ND, McLellan TA, Cotto JH, Karithanom M, Weiss SR. Characteristics of opioid prescriptions in 2009. JAMA. 2011;305: 1299-301.

5. Hoppe JA, Nelson LS, Perrone J, Weiner SG. Opioid prescribing in a cross section of US emergency departments. Ann Emerg Med. 2015;66:253-9.e1

6. Menchine MDAS, Plantmason L, et al. Strength and dose of opioid prescribed from US emergency departments compared to office practices: implications for emergency department safe-prescribing guidelines. Ann Emerg Med. 2014;64.

7. Butler MM, Ancona RM, Beauchamp GA, et al. Emergency department prescription opioids as an initial exposure preceding addiction. Ann Emerg Med 2016.

8. Baehren DF, Marco CA, Droz DE, Sinha S, Callan EM, Akpunonu P. A statewide prescription monitoring program affects emergency 
department prescribing behaviors. Ann Emerg Med. 2010;56:19$23 . \mathrm{e} 1-3$

9. Kilaru AS, Gadsden SM, Perrone J, Paciotti B, Barg FK, Meisel ZF. How do physicians adopt and apply opioid prescription guidelines in the emergency department? A qualitative study. Ann Emerg Med. 2014;64:482-9 .e1

10. Weiner SG, Raja AS. Bittner JC, et al. Acad Emerg Med: Opioidrelated Policies in New England Emergency Departments; 2016.

11. Beaudoin FL, Banerjee GN, Mello MJ. State-level and system-level opioid prescribing policies: the impact on provider practices and overdose deaths, a systematic review. J Opioid Manag. 2016;12: 109-18.

12. Garg RK, Fulton-Kehoe D, Turner JA, et al. Changes in opioid prescribing for Washington workers' compensation claimants after implementation of an opioid dosing guideline for chronic noncancer pain: 2004 to 2010. J Pain. 2013;14:1620-8.

13. Fox TR, Li J, Stevens S, Tippie T. A performance improvement prescribing guideline reduces opioid prescriptions for emergency department dental pain patients. Ann Emerg Med. 2013;62:237-40.

14. Pomerleau AC, Nelson LS, Hoppe JA, Salzman M, Weiss PS, Perrone J. The impact of prescription drug monitoring programs and prescribing guidelines on emergency department opioid prescribing: a multi-center survey. Pain Med 2016.
15. Patel MS, Day SC, Halpern SD, et al. Generic medication prescription rates after health system-wide redesign of default options within the electronic health record. JAMA Intern Med. 2016;176:847-8.

16. Stanek JJ, Renslow MA, Kalliainen LK. The effect of an educational program on opioid prescription patterns in hand surgery: a quality improvement program. J Hand Surg [Am]. 2015;40:341-6.

17. del Portal DA, Healy ME, Satz WA, McNamara RM. Impact of an opioid prescribing guideline in the acute care setting. J Emerg Med. 2016;50:21-7.

18. Tamayo-Sarver JH, Dawson NV, Cydulka RK, Wigton RS, Baker DW. Variability in emergency physician decision making about prescribing opioid analgesics. Ann Emerg Med. 2004;43:483-93.

19. Gibbons RV, Landry FJ, Blouch DL, et al. A comparison of physicians' and patients' attitudes toward pharmaceutical industry gifts. J Gen Intern Med. 1998;13:151-4.

20. DeJong C, Aguilar T, Tseng CW, Lin GA, Boscardin,WJ, Dudley RA. Pharmaceutical industry-sponsored meals and physician prescribing patterns for medicare beneficiaries. JAMA Intern Med 2016.

21. Grande D, Frosch DL, Perkins AW, Kahn BE. Effect of exposure to small pharmaceutical promotional items on treatment preferences. Arch Intern Med. 2009;169:887-93. 